\title{
Lumen
}

Selected Proceedings from the Canadian Society for Eighteenth-Century Studies

\section{Adèle de Sénange (1794) et sa réception}

\section{Marie-France Silver}

Volume 14, 1995

URI : https://id.erudit.org/iderudit/1012513ar

DOI : https://doi.org/10.7202/1012513ar

Aller au sommaire du numéro

Éditeur(s)

Canadian Society for Eighteenth-Century Studies / Société canadienne d'étude du dix-huitième siècle

ISSN

1209-3696 (imprimé)

1927-8284 (numérique)

Découvrir la revue

Citer cet article

Silver, M.-F. (1995). Adèle de Sénange (1794) et sa réception. Lumen, 14, 119-126. https://doi.org/10.7202/1012513ar d'utilisation que vous pouvez consulter en ligne.

https://apropos.erudit.org/fr/usagers/politique-dutilisation/ 


\section{Adèle de Sénange (1794) et sa réception}

L'étude des circonstances entourant la publication d'Adèle de Sénange offre un nouvel exemple des efforts des Françaises émigrées sous la Révolution pour survivre. Étudier la réception de ce roman éclaire aussi l'évolution de la sensibilité du XVIII ${ }^{\mathrm{e}}$ siècle finissant, tout comme le développement du roman féminin post-révolutionnaire. ${ }^{1}$

Dans Trois Femmes d'Isabelle de Charrière, paru en 1795, les deux jeunes héros, Théobald et Émilie, discutent avec animation Adèle de Sénange qu'ils décrivent comme une 'nouveauté charmante ... que tout le monde a lue, que tout le monde a admirée. ${ }^{2}$

Paru en 1794 à Londres, Adèle de Sénange ou Lettres de Lord Sydenham de la comtesse de Flahaut, ${ }^{3}$ future Madame de Souza, connut en effet un succès immédiat tant chez les aristocrates que chez les révolutionnaires. Ce roman fut réimprimé à Hambourg en 1797, à Paris en $1798^{4}$ et de multiples fois au cours du XIX ${ }^{\mathrm{e}}$ siècle. Il inspira à Scribe sa comédievaudeville $L a$ Pensionnaire mariée qui fut représentée pour la première fois à Paris en 1835.

Sous la Terreur, le comte de Flahaut fut arrêté puis guillotiné. Madame de Flahaut, privée de tous ses biens, se réfugia à Londres avec son fils et dut, comme tant d'autres émigrées, trouver des moyens de subsistance. Elle avait emporté dans ses bagages un manuscrit commencé au début de la Révolution et qu'elle décida de terminer et de publier. ${ }^{5} \mathrm{Au}$ printemps 1793 elle en fit des lectures dans les salons londoniens. ${ }^{6} \mathrm{Mme}$ de Flahaut, qui jadis avait reçu ses amis dans son somptueux salon du Louvre, les recevait maintenant dans les chambres qu'elle louait au-dessus d'une épicerie. Dans sa correspondance, Sir Gilbert Elliot, first Earl of Minto, rappelant la soirée de mars 1793 où il avait rencontré Mme de Staël chez Mme de Flahaut, écrivit, '[W] sat in a miserable lodging, over a grocer's [talking] till two o'clock."

Espérant donc quelque argent de son roman, la comtesse prépara son manuscrit. Évoquant cette période, elle devait écrire plus tard:

Je me souviens qu'étant en Angleterre une pauvre émigrée et voulant y faire imprimer Adèle de Sénange je fus obligée, n'ayant pas de quoi payer un copiste, 
de récrire tout mon manuscrit, avec le soin de mettre les points, les virgules, les grandes lettres, enfin toutes les exactitudes qui n'allaient pas trop à mon caractère à l'âge que j'avais alors. ${ }^{8}$

Dans le cercle des émigrés français de Londres, le succès fut immédiat. Dans une lettre du 26 avril 1794, le général de Montesquiou, évoquant l'accueil du roman à Londres, commente: 'Il a eu beaucoup de succès auprès du petit nombre de personnes qui a été porté à le lire, entr' autres Madame de Staël. ${ }^{9}$

Vendu par souscription, Adèle de Sénange mit Mme de Flahaut à l'abri du besoin pendant quelques années et lui permit ainsi de pourvoir à l'éducation de son fils. Ce succès financier frappa les contemporains. 'Avez-vous Adèle de Sénange joli roman d'une émigrée, Mme de Flaho [sic]?' demande Isabelle de Charrière à une amie; et elle ajoute, 'Cela a été écrit et imprimé par souscription en Angleterre et a valu beaucoup d'argent à son auteur. ${ }^{10}$ En 1836, à la mort de Mme de Souza, le Journal des Débats rappela le succès d'Adèle de Sénange en ces termes: 'toute l'aristocratie du royaume qui goûtait [la] conversation [de son auteur] souscrivit pour son ouvrage, et près de 40000 francs devinrent le prix de cette souscription. Le succès littéraire fut immense. ${ }^{11}$ Double succès, donc, à la fois littéraire et financier.

Ce petit roman épistolaire est basé sur une intrigue très simple: l'amour d'Adèle de Sénange et de Lord Sydenham, amour d'abord interdit car Adèle est mariée, puis autorisé puisqu'elle devient veuve. Sainte-Beuve, qui voyait en Adèle de Sénange une soeur de la princesse de Clèves, décrit le roman comme l'histoire de la

passion croissante, qui se dérobe ... sous des rideaux de verdure et se replie en délicieuses lenteurs; des orages passagers, sans ravages, semblables à des pluies d'avril; la plus difficile des situations honnêtes menée à la fin jusque dans ses moindres alternatives, avec une aisance qui ne penche jamais vers l'abandon, avec une noblesse de ton qui ne force jamais la nature, avec une mesure indulgente pour tout ce qui n'est pas indélicat: tels sont [conclut-il] les intérêts principaux d'un livre où pas un mot ne rompt l'harmonie. ${ }^{12}$

Très habilement, à la peinture de l'amour naissant d'Adèle pour Sydenham, la romancière joint celle de l'affection filiale d'Adèle pour son vieux mari, et celle du développement de l'amitié qui va s'établir entre Sydenham et M. de Sénange. Sydenham, qui croit en effet au début qu'Adèle s'est laissée 'acheter' par M. de Sénange, découvre par la suite la générosité de ce dernier qui, en épousant Adèle, a sauvé la jeune fille du cloître où voulait l'enfermer sa mère; Sydenham découvre aussi, peu à peu, l'affection réelle et mutuelle des deux époux. La romancière ex- 
plore, sans aucun jugement moral, les liens affectifs qui se tissent lentement, et comme malgré eux, entre les quatre personnages. Ces derniers vont inspirer d'autres romancières: l'affection d'Adèle pour $M$. de Sénange qu'elle a épousé à seize ans est parallèle à celle que Delphine, dans le roman de Mme de Staël paru en 1802, éprouve pour M. d'Albermale qui l'a épousée à seize ans pour la sauver de la pauvreté. Élevée au couvent, Adèle n'a pas acquis les manières factices de la vie mondaine et garde un enthousiasme qui charme son entourage. Sophie Cottin s'en inspira pour créer le personnage de Frédéric, le héros de Claire d'Albe. Frédéric, lui, n'a pas été élevé au couvent mais dans les Cévennes. Le couvent dans un cas, les Cévennes dans l'autre, permettent aux deux auteurs d'innocenter l'amour naissant, par l'ignorance où se trouvent Adèle et Frédéric d'identifier le sentiment qui les anime. Cette vie, à l'écart du monde, leur donne à tous deux une spontanéité et une fraîcheur qui, en les démarquant de leur entourage, va faire naître l'intérêt de Sydenham pour Adèle comme celui de Claire pour Frédéric. Adèle est un intéressant portrait de femme, celui d'une adolescente lumineuse, à la fois égoïste et affectueuse, sensible et enthousiaste, incapable de se soustraire à l'emprise d'une mère qui pourtant ne l'aime pas; lucide sur sa propre faiblesse elle explique ainsi à Sydenham ses hésitations à l'épouser après la mort de son mari: 'Je ne suis assez forte ni pour déplaire à ma mère, ni pour vous fâcher. ${ }^{, 13}$

Delphine sera elle aussi tiraillée entre son inclination pour Léonce et l'opposition de Mme de Vernon. Mme de Flahaut semble avoir relancé, par le succès de son livre, le thème de l'amour interdit. On retrouve en effet une intrigue tout-à-fait identique dans Claire d'Albe et très similaire dans les Voeux téméraires de Mme de Genlis et dans Delphine. Théobald, le héros du roman d'Isabelle de Charrière dont nous avons déjà parlé, cite Sydenham et voit dans Adèle de Sénange l'expression de ses propres sentiments pour Émilie. ${ }^{14}$

Ironiquement, cette expression du sentiment amoureux qui impressionna les contemporains de Mme de Flahaut et la génération romantique est tournée en dérision par l'auteur elle-même. Au début du roman en effet, une amie de Sydenham amuse le jeune homme en lui racontant un conte de fées. L'auteur indique simplement dans une note qu'elle joint ce dernier en appendice au roman. Si l'on s'en tient à la seule lecture du roman comme l'ont fait les romantiques, l'intrigue amoureuse a toutes les qualités d'une sensibilité romantique et l'on finit le roman sur la vision d'Adèle ayant trouvé le bonheur dans son amour pour Sydenham. Si on lit l'appendice, comme l'a désiré l'auteur en octroyant cette place au conte, on y trouve une très spirituelle critique des femmes qui consacrent leur vie à un homme, et qui misent tout sur l'amour. ${ }^{15}$ L'ensemble pourrait alors être interprêté comme une satire de la valori- 
sation romantique de l'amour puisque l'auteur nous fait ainsi comprendre que l'amour qu'Adèle voue à Sydenham, loin de la conduire au bonheur, pourrait au contraire la détruire.

Mais qu'ils se soient ou non mépris sur les intentions de l'auteur, les contemporains admirèrent énormément le roman. La sobriété de son expression fut relevée comme un trait particulièrement original par une génération nourrie d'éloquence révolutionnaire. Marie-Joseph Chénier admire dans Adèle de Sénange 'un style orné avec mesure, la condition d'un bon livre et l'aisance d'une conversation fleurie; l'esprit qui ne dit rien de vulgaire, le goût qui ne dit rien de trop. ${ }^{\prime 16}$ Le roman de Mme de Flahaut contraste en effet vivement avec le roman noir alors à la mode. Legouvé remarque: 'Il parut dans un temps où l'on était inondé des sombres productions des romanciers anglais qui croient plaire avec des spectres et des horreurs, et comme il n'a rien d'un si lugubre appareil, comme les ressorts en sont simples, il repose agréablement des compositions tristes et convulsives. ${ }^{17}$

Mais paradoxalement, le succès du roman de Mme de Flahaut allait encourager les femmes écrivains de la fin du siècle à privilégier certains motifs funèbres. A sa mort, M. de Sénange est en effet enterré au bord d'une rivière, sous des peupliers, dans la petite île du parc de sa maison de campagne. Cette île était la retraite préférée d'Adèle, l'Élysée' où, comme Julie, elle ne recevait que les êtres qui lui étaient chers. Veuve, Adèle est libre d'épouser Sydenham et les amants se recueillent sur la tombe de $M$. de Sénange où Adèle fait graver ce vers: 'On ne me répond pas, mais peut-être on m'entend.'

C'est sur ce même alexandrin que Mme de Staël choisit de terminer Delphine. Il est gravé sur un arbre, près du tombeau qui a enfin réuni Delphine et Léonce, tombeau qui se trouve lui aussi au bord d'une rivière sous des peupliers. Le motif du tombeau est également présent dans Claire d'Albe, roman de Mme Cottin, qui date de 1799, et dans les Voeux téméraires de Mme de Genlis, paru la même année. Dans Claire d'Albe, l'héroïne dirige souvent ses promenades vers la tombe de son père, et c'est sur cette dernière - Freud se serait réjoui s'il avait lu le roman que Claire va enfin se donner à Frédéric, avant, bien entendu, d'expirer. Dans les Voeux téméraires, Constance, jeune Anglaise exilée en France, n'a donc pas pu emporter avec elle le tombeau de son mari mais - qu'à cela ne tienne - elle en a fait faire un tableau qu'elle emmène partout avec elle et sur lequel elle médite souvent. Discret dans Adèle de Sénange, le motif du tombeau prend une place disproportionnée dans les romans féminins postérieurs, se transformant en topos caractéristique du premier romantisme.

Sainte-Beuve a très bien vu que la force du roman de Mme de Flahaut tenait pourtant justement au fait qu'il échappait au romanesque et au 
sentimental. Il loue la romancière d'y avoir présenté 'la vie réelle dans sa plus fine véritée. ${ }^{18}$ Avec Adèle de Sénange, Mme de Flahaut a en effet créé un roman intimiste, le premier peut-être de notre littérature. Dans son Avant-propos, qui est une fort intéressante réflexion sur le développement du genre romanesque, et qui précède d'un an l'Essai sur les fictions de Mme de Staël, Mme de Flahaut critique les romans de Richardson. Tout en reconnaissant l'apport positif du roman sentimental qui a engagé la littérature dans l'étude de la vie privée, la romancière souhaite en effet un roman moins 'grand', moins 'élevé', moins didactique.

J'aime à croire que l'on pourrait se rapprocher davantage de la nature, et l'on ne manquerait pas à l'utilité, peut-être même à l'intérêt, en cherchant à tracer ces détails fugitifs qui occupent l'espace entre les événements de la vie. Des jours, des années dont le souvenir est effacé, ont été remplis d'émotions, de sentiments, de petits intérêts, de nuances fines et délicates: chaque moment a son occupation, et chaque occupation a son ressort moral: il est même bon de rapprocher, sans cesse, la vertu de ces circonstances obscures et inaperçues, parce que c'est la suite de ces devoirs journaliers qui forme essentiellement le fond de la vie. Ce sont ces ressorts que j'ai taché de démêler. ${ }^{19}$

N'est-ce pas là, exprimé, ce que Sainte-Beuve essaiera - sans succès d'ailleurs - de montrer dans son roman Volupté? Il y aurait probablement une fort intéressante étude à faire sur l'influence de Mme de Flahaut sur l'auteur de Volupté.

Le charme élusif du roman de Mme de Flahaut n'avait pas échappé à Isabelle de Charrière. Cette dernière qui, impressionnée par le succès $\mathrm{d}$ 'Adèle de Sénange, avait entrepris son roman Trois femmes, remarquait en effet: 'Je doute que cela plaise autant qu'Adèle de Sénange dans laquelle il y a je ne sais quelle grâce légère et volatile toute particulière..${ }^{20}$ Le charme du roman de Mme de Flahaut tient peut-être à la nostalgie discrète qui s'en dégage. Adèle de Sénange est en effet le roman d'une double nostalgie: nostalgie d'Adèle pour le monde de l'enfance, nostalgie de l'auteur pour un monde innocent. Pour Adèle 'la vie se partage en deux époques, un couvent où [elle] a été élevée dans le bonheur durant des années, un mariage heureux encore, mais inégal par l'âge ${ }^{\prime 21}$ et sans passion. Le souvenir lumineux du couvent qu'Adèle garde précieusement est fort probablement l'écho de l'attachement de l'auteur pour ses années au couvent. Ses biographes, Sainte-Beuve et Maricourt, en sont en tout cas convaincus. Il est certain que l'institution du couvent, généralement décriée dans les romans de la période, est présentée très positivement dans Adèle de Sénange et dans deux autres des romans de l'auteur. ${ }^{22}$ De plus, le besoin qu'Adèle éprouve de se trouver des asiles - que ce soit le couvent de sa jeunesse - ou la petite île du parc de Neuilly dans sa vie 
adulte - trahit une angoisse face à la réalité. C'est ce même désir de se détourner du réel qui a poussé la comtesse de Flahaut à écrire, comme elle l'explique fort clairement dans son Avant-propos: 'Cet essai a été commencé dans un temps qui semblait imposer à une femme, à une mère, le besoin de s'éloigner de tout ce qui était réel ....' ${ }^{23}$

L'écriture fournit donc à l'auteur un refuge contre les événements extérieurs. Aucun écho de ces derniers ne retentit en effet dans le roman dont l'intrigue de déroule quelques années à peine avant la Révolution. Les flâneries dans les parcs, les promenades sur l'eau, les rêveries sur l'île, les causeries railleuses ou tendres, gaies ou mélancoliques revêtent alors une qualité de rêve qui dût se communiquer au lecteur des années 1790 avec une acuité particulière. On pourrait suggérer alors une troisième nostalgie: celle du lecteur pour une civilisation qu'il sait disparue. Sainte-Beuve ne s'y est pas trompé. Il disait en effet trouver dans Adèle de Sénange des 'parfums du passé, d'un passé d'hier encore, mais qui ne se retrouvera plus.' Et il ajoutait:

Les peintures nuancées dont nous parlons supposent un goût et une culture d'âme que la civilisation démocratique n'aurait pas abolis sans inconvénient pour elle-même, s'il ne devait renaître dans les mœurs nouvelles quelque chose $\mathrm{d}^{\prime}$ analogue un jour. ${ }^{24}$

Il y a lieu de souligner que le refus formulé par Mme de Flahaut de laisser l'actualité s'immiscer dans la fiction n'est pas justifié par des raisons de convenance, d'étiquette, mais bien par un choix tout-à-fait personnel. Ce refus répondant aux mêmes motivations, fut réitéré en 1799 par Sophie Cottin dans la préface de Claire d'Albe ${ }^{25}$ et par Mme de Staël dans une lettre datée du premier octobre 1800 où elle se justifiait de donner à la Révolution un rôle mineur, voire inexistant, dans Delphine qu'elle était alors en train de rédiger. ${ }^{26}$

Loin donc d'avoir incité les femmes à renouveler le roman féminin en y incorporant des questions d'actualité, la Révolution semble au contraire les avoir confirmées dans leur rejet de l'ingérence du politique dans la fiction. Isabelle de Charrière, seule, à cette époque, $n$ 'hésita pas à délaisser les formes traditionnelles du roman féminin pour ouvrir le roman au domaine politique. Mais avec Adèle de Sénange qui est son premier grand succès, le roman féminin post-révolutionnaire s'engage pourtant sur une voie nouvelle. Se démarquant du roman sentimental de Richardson, le roman de Mme de Flahaut propose un nouveau genre de roman qui se nourrit cependant lui aussi de la vie privée: le roman intimiste. Il serait donc justifié de l'inclure dans les études relatives au développement du genre romanesque. Le roman de Mme de Flahaut influença d'autre part des romancières aussi différentes qu'Isabelle de 
Charrière, Mme de Staël, Sophie Cottin et même Mme de Genlis. A ce titre aussi, il mérite d'être tiré de l'oubli.

\section{MARIE-FRANCE SILVER \\ Collège Glendon, Université York.}

\section{Notes}

1 Pour une comparaison détaillée des romans féminins de la décade révolutionnaire, voir Marie-France Silver, 'Le roman féminin des années révolutionnaires', Eighteenth-Century Fiction 6 (1994). Ce qui y est dit de Mme de Flahaut est largement inspiré du présent article.

2 Isabelle de Charrière, Trois femmes, Oeuvres complètes (Amsterdam: G. A. Van Oorschot, 1981), 2:77.

3 Adelaïde-Marie-Émilie Filleul, comtesse de Flahaut, puis marquise de Souza (1761-1836).

4 Je n'ai pas pu vérifier l'existence de cette édition, qui est attestée par Michaud, Biographie universelle (Paris: Delagrave, 1855), 39:723-24.

5 Le Baron André de Maricourt, Madame de Souza et sa famille (Paris: Emile-Paul, 1907).

6 Mme de Staël-Holstein, Correspondance générale, vol. 3, pt. 1 ([Paris]: J.-J.Pauvert, 1968) $99-100$, n. 1.

7 Ibid. (1965), vol. 2, pt. 2, 409, n. 2.

8 Cité par Maricourt, 3.

9 Staël-Holstein, vol. 3, pt. 1, 100, n. 1.

10 Charrière, lettre à Henriette L'Hardy des 10-14 février 1795, citée dans Préface de Trois Femmes 24.

11 Cité par Maricourt, 175.

12 Sainte-Beuve, notice sur Mme de Souza et ses ouvrages, dans Oeuvres de Mme de Souza (Paris: Charpentier, 1845).

13 Mme de Flahaut, Adèle de Sénange (Hambourg, 1797) 161.

14 Charrière, Trois Femmes 77.

15 Adèle de Sénange, appendice ('Aglae, conte') 201-7.

16 Cité par Sainte-Beuve, 77.

17 Cité par Michaud, 723.

18 Sainte-Beuve, 15.

19 Adèle de Sénange, Avant-Propos, xv-xvi.

20 Isabelle de Charrière, lettre à Henriette L'Hardy des 10-14 février 1795 (n. 10) 24.

21 Sainte-Beuve, 8.

22 Eugénie et Matilde et La comtesse de Fargy. 
126 Marie-France Silver

23 Adèle de Sénange, Avant Propos, xi-xii.

24 Sainte-Beuve, 2.

25 Sophie Cottin, Claire d'Albe (Paris: Régine Deforges, 1976).

26 Mme de Staël, lettre à Hochet datée du $1^{\mathrm{er}}$ octobre 1800 , citée dans l'introduction à Delphine (Genève: Droz, 1990) 1:15. 